\title{
Veso: virtual earth-sun observatory
}

\author{
G. Cifuentes-Nava • J. A. González-Esparza • \\ E. Hernández-Quintero • A. Lara-Sánchez • \\ J. F. Valdés-Galicia
}

Received: 10 September 2007 / Accepted: 10 January 2008 / Published online: 21 February 2008

(C) Springer-Verlag 2008

\begin{abstract}
We present the Virtual Earth-Sun Observatory (VESO) at the web site http://www.veso.unam.mx. This site shows a real time integrated database obtained from four instruments of the Instituto de Geofisica-UNAM studying Sun-Earth connection phenomena. (1) The Solar Radio Interferometer (RIS, Radio Interferómetro Solar) measures the lower solar atmosphere radiation at $7.5 \mathrm{GHz}$, revealing microwave bursts associated with solar activity. (2) The Mexican Array Radio Telescope (MEXART, Observatorio de Centelleo Interplanetario de Coeneo) will detect solar wind large-scale disturbances between Sun and Earth (e.g., Interplanetary counterparts of Coronal Mass Ejections (ICMES) and Stream Interaction Regions (SIR)) employing the interplanetary scintillation technique (IPS) operating at 140 MHz. (3) The Cosmic Ray Observatory (RC) detects high energy galactic particles, whose flow is affected by magnetic disturbances in the solar wind, and (4) the Teoloyucan Geomagnetic Observatory (TEO) measures the variations in the Earths magnetic field. The VESO instruments provide data from four different points of the complex chain of the solar terrestrial relations and will
\end{abstract}

\footnotetext{
Communicated by P. Fox

J. A. González-Esparza $(\bowtie)$

Instituto de Geofísica,

Universidad Nacional Autónoma de México,

Tzitnzuntzan 310, Vista Bella,

58098 Morelia, Mich., Mexico

e-mail: americo@geofisica.unam.mx

G. Cifuentes-Nava • E. Hernández-Quintero • A. Lara-Sánchez •

J. F. Valdés-Galicia

Instituto de Geofísica,

Universidad Nacional Autónoma de México,

Ciudad Universitaria,

Coyoacan Mexico DF 04510, Mexico
}

allow the study of intense solar events causing geomagnetic activity. The VESO project is part of the celebration of the International Heliophysical Year (IHY) and the Electronic Geophysical Year (EGY) in Mexico.

Keywords Earth · Sun · Observatory

\section{Introduction}

On February 19th, 2007, the United Nations Organization initiated formally the celebration of the International Heliophysical Year (IHY). This worldwide celebration combines efforts of countries all over the world in order to study the Sun and its influence in Heliosphere and in our planet. The main goals of the IHY are: to develop the basic science of heliophysics through cross-disciplinary studies of universal processes; to determine the response of terrestrial and planetary magnetospheres and atmospheres to external drivers; to foster international scientific cooperation in the study of heliophysical phenomena now and in the future; and to communicate unique IHY results to the scientific community and the general public (Harrison et al. 2005). On the other hand, the Electronic Geophysical Year (eGY) provides a frame for the international geoscientific community to open access to data, data preservation, data discovery, data rescue, capacity building, and outreach (Rex 2007). The development of Virtual Observatories and Laboratories is one of the main goals of the eGY.

\section{The Virtual Earth-Sun Observatory (VESO)}

Solar activity produces perturbations traveling in the interplanetary medium that may interact with the Earth 
Table 1 The VESO instruments

\begin{tabular}{lll}
\hline Instrument & Location & Data \\
\hline RIS & Mexico City & $\begin{array}{c}\text { Microwave radiation flux of the lower solar atmosphere }(7.5 \mathrm{GHz}), 8 \mathrm{~h} \text { of daily } \\
\text { operation, } 4 \text { channels } / 20 \mathrm{samples} \text { per second, each size per channel: } 500 \mathrm{~Kb}\end{array}$ \\
MEXART & Coeneo, Michoacan & $\begin{array}{c}\text { Electromagnetic flux at } 140 \mathrm{MHz}, 24 \mathrm{~h} \text { operation, } 64 \text { channels sampling } 100 \text { times } \\
\text { per second, size per channel: } 14 \mathrm{Mb}\end{array}$ \\
CR & Fexico City & $\begin{array}{c}\text { Flux of cosmic ray nucleonic component } 24 \mathrm{~h} \text { operation sampling: } 1 \text { channel } 1 \text { sample } \\
\text { every } 5 \text { min size per channel: } 15 \mathrm{~Kb}\end{array}$ \\
TEO & Teoloyucan, Edo. Mexico & $\begin{array}{c}\text { Vector geomagnetic field operation: } 24 \mathrm{~h} \text { sampling } 7 \text { channels } 12 \text { times per minute } \\
\text { size per channel: } 100 \text { Kbytes }\end{array}$ \\
\hline
\end{tabular}

magnetic field and induce severe variations known as geomagnetic storms. Nowadays it is known that a great variety of telecommunication systems, transformers, power lines, pipelines, satellites, spacecrafts control, etc, may be damaged because this solar activity. The study of solar perturbations traveling to Earth, have reached great importance and the term "Space Weather" was coined to describe the state of perturbation on the interplanetary medium and the magnetic behavior around Earth. As a part of these studies running by the international community, the Instituto de Geofísica of the Universidad Nacional Autónoma de México (UNAM) is undertaking the project to join four observational devices to shape a Virtual Earth Sun Observatory (VESO) to measure the explosive activity in Sun's surface, Coronal Mass Ejections (CMEs) and Stream Interaction Regions (SIR) in the interplanetary medium, Cosmic Rays that reach Earth, and the Magnetic Field perturbations in our planet. Such instrumentation will participate actively, in collaboration with other observatories and spacecrafts, conforming a worldwide monitoring of the Earth-Sun environment. The VESO project is part of the participation of Mexico in the IHY and eGY efforts.

Table 1 summarizes the main characteristics of the four VESO instruments. The instruments are located at different sites. The following sections describe the instruments.

\section{Solar Radio Interferometer (RIS)}

Figure 1 shows the Solar Radio Interferometer (RIS; http:// cintli.igeofcu.unam.mx/index.html) which is a Sun observing telescope in centimeter wavelengths $(4 \mathrm{~cm})$. The RIS was manufactured in USSR, nevertheless, in the last years has been subject to a total renovation. The RIS records microwave solar emission during eight hours every day, when the solar disk is visible from the site. This emission is approximately constant (the so called quiet Sun emission) except when a Solar flare takes place (an eruptive event in the low solar Corona). Flares are the first indicators of solar activity and usually are accompanied by a Coronal
Mass Ejection; both phenomena drive the Space Weather. RIS measures the total and polarized flux as well as the relative position of the flare source in the North-South direction.

\section{Mexican Array Radio Telescope (MEXART)}

The MEXART (http:/www.mexart.unam.mx/) main scientific objective is to track large-scale solar wind perturbations (such as CMEs and SIRs) in its course from the Sun to the Earth using the Interplanetary Scintillation (IPS) technique (Fig. 2). The MEXART antenna is composed of a 4,096 (64X64) array dipole-kind antennas reaching a total physical area of $9,800 \mathrm{~m}^{2}$. The East-West length of this array is $140 \mathrm{~m}$, and North-South is $80 \mathrm{~m}$ (see details in Gonzalez-Esparza et al. 2004). The MEXART is a transit radio telescope configured to detect extragalactic radio sources which signal is affected by solar wind electron density variations. To date it is undergoing its final stage of calibration (Gonzalez-Esparza et al. 2005, 2006). Table 2 presents the summary of the array technical features.

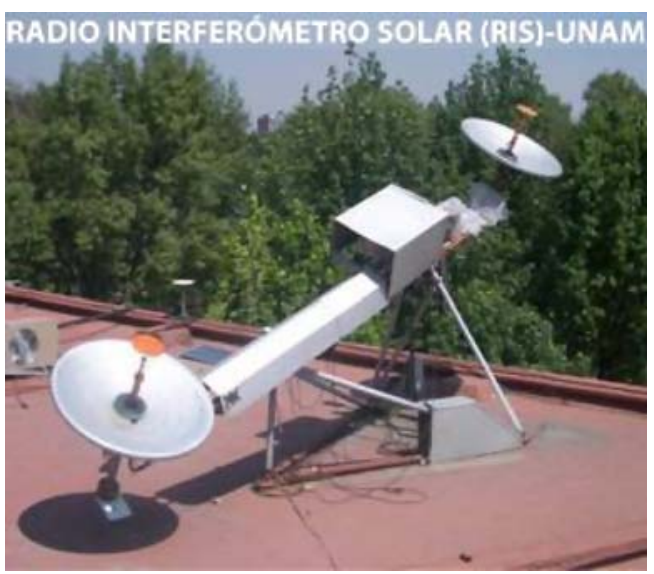

Fig. 1 The RIS consists of two antennas of $1 \mathrm{~m}$ of diameter in an equatorial mount and separated by 120 wavelengths 


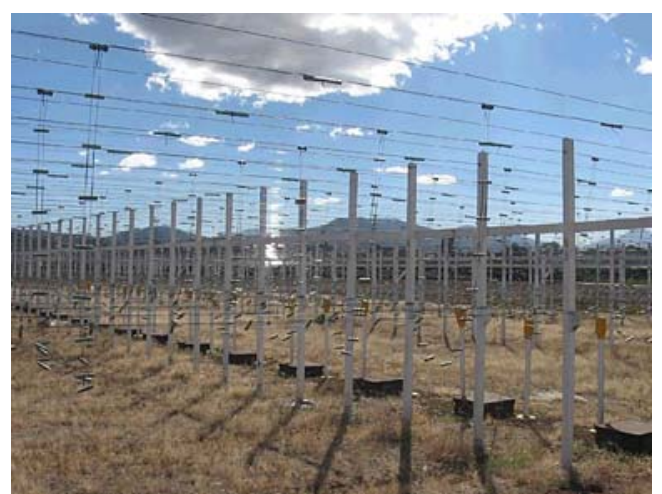

Fig. 2 The dipoles array at MEXART

\section{Cosmic Ray Observatory (RC)}

From all possible directions the Earth is influenced by an obstinate particle shower from cosmic far away sites. This shower is known as Cosmic Rays (CR). CR are space traveling mostly charged energetic particles, some of these are capable to cross the Earth magnetosphere and atmosphere reaching places at and deep under its surface. The study of CR is very important since they are the only material information available arriving from astrophysical sites beyond Earth, the variety in its chemical composition have high significance to figure out astrophysical enigmas not yet solved, its energies cover a wide interval ranging from binding nuclei forces, to those sensible to our day to day life. The lowest CR's energies $\left(E<10^{9} \mathrm{eV}\right)$ are controlled by the Sun's cavity (Heliosphere). The cosmic radiation detected in the Earth's surface is influenced by its magnetic field and atmosphere. CR travel through the Heliosphere where solar wind pushes them outward and the interplanetary magnetic field drives them inward. Changes in solar wind conditions (e.g. a CME), cause variations in $\mathrm{CR}$ intensity. By means of a detector known as Neutron Monitor (Hatton and Carmcheal 1964) the RC Observatory continuously tracks CR flux (http://www.geofisica.unam. $\mathrm{mx} /$ isyp/orc/) (Fig. 3) with energies greater than $8.2 \times$ $10^{9} \mathrm{eV}$. The lowest detectable energy is given by the presence of the geomagnetic field that acts as a magnetic spectrometer of the charged CR.

Table 2 MEXART characteristics

\begin{tabular}{ll}
\hline Parameter & Characteristic \\
\hline Central operation frequency & $139.65 \mathrm{MHz}$ \\
Bandwidth & $1.5 \mathrm{MHz}$ \\
Basic Element & Full-wave dipole \\
Number of Elements & 4,096 \\
Number of lines East-West & 64 with 64 dipoles per line \\
Number of receptor & $1-2$ \\
Number of Butler matrixes & 2, each with 32 ports \\
\hline
\end{tabular}

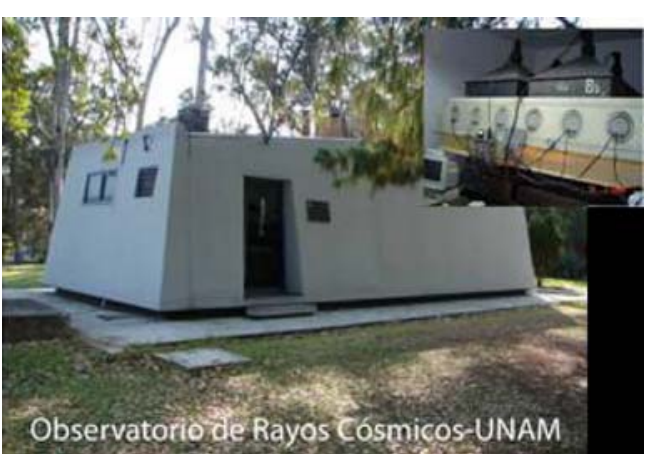

Fig. 3 The Cosmic ray observatory at the UNAM Campus in Mexico City. The inset is a photo of the six proportional counters of the Neutron Monitor, the black boxes are part of a muon telescope currently under renovation

\section{Teoloyucan Geomagnetic Observatory (TEO)}

Geomagnetic field variations are caused by internal and external sources. The Worldwide Geomagnetic Observatories Network (INTERMAGNET) measures and detects diverse external magnetic features, mainly coming from the Sun and driven by interplanetary disturbances. Geomagnetic storms, flares, bays, sudden storm commencements, diurnal variations, and the level of geomagnetic activity are some of the complex phenomena reported by this network. The Teoloyucan Geomagnetic Observatory (operating since 1914) (Fig. 4) (http://132.248.6.186/ OMTeoloyucanIN.html) is part of the INTERMAGNET network since 2002. Its participation in the VESO project will allow us to perform global analysis of potential geoeffective solar storms driving interplanetary disturbances. The TEO geomagnetic measurements will close the

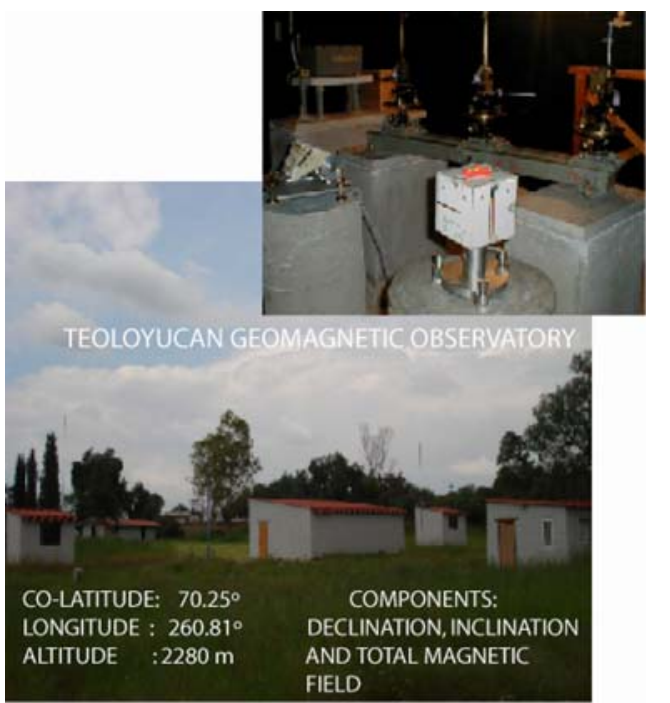

Fig. 4 Geomagnetic position, place and main instrumentation in Teoloyucan 
Fig. 5 VESO flux diagram

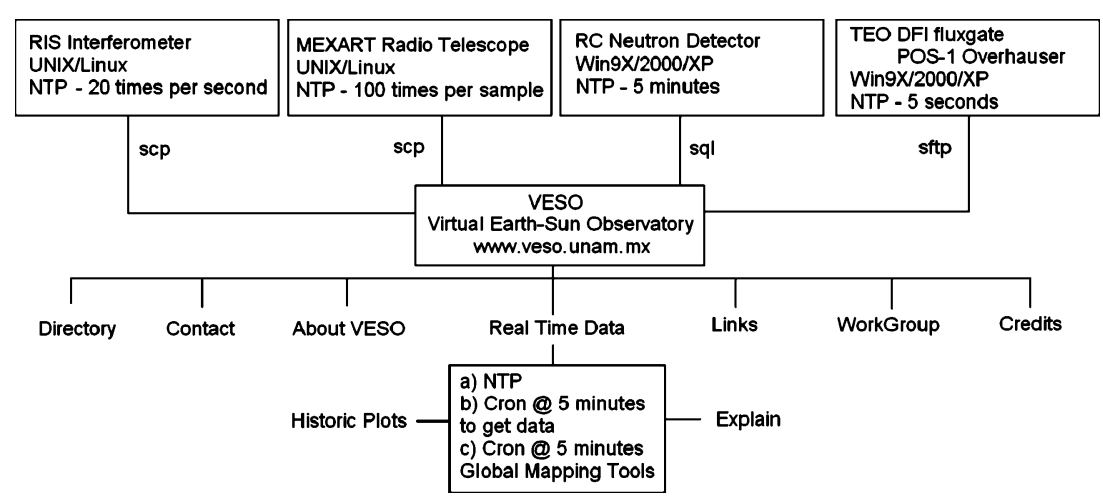

complex chain of solar terrestrial phenomena track by the VESO instruments.

\section{Veso operation}

The VESO integrates data in real time from four instruments with different operation system and data production. Figure 5 shows the flux diagram of the VESO operation. RIS samples 20 times per second with four channels under Linux platform using a protocol scp for data transferring. MEXART samples 100 times per second with one channel (eventually it will use 64 channels) under Linux platform using a protocol scp for data (compressed) transmission. RC samples flux of neutrons once every five minutes combining platforms Win9X/2000/XP and Linux storing in sql for transmitting the data. TEO samples magnetic field with seven channels 12 times per minute, the system POS-1 Overhauser produces a data file combining platforms
Win9X/2000/XP and Linux and it uses sftp for transmitting the data.

\section{Veso observations}

Figure 6 shows an example of a real time daily plot at the VESO: the measurements of the four instruments versus universal time (UT). The upper plot shows the RIS record of the solar radio emission at $7.5 \mathrm{GHz}$ (the abrupt fall in the plot is a calibration signature). Next below, is the MEXART scanning of the radio sky at $140 \mathrm{MHz}$. The curve shows the radio trace of the Galaxy, where the big peak is the transit of the Galaxy nucleus and the sharp peaks are cosmic radio sources. Next below there is the flux of cosmic rays measurements at RC in Mexico City, which is affected by interplanetary disturbances. At the bottom of the figure is the Earth magnetic field intensity as detected by TEO. In principle, a geoeffective solar storm could be initiated by a
Fig. 6 Real time measurements at the VESO server
VESO UNAM Preliminary Data

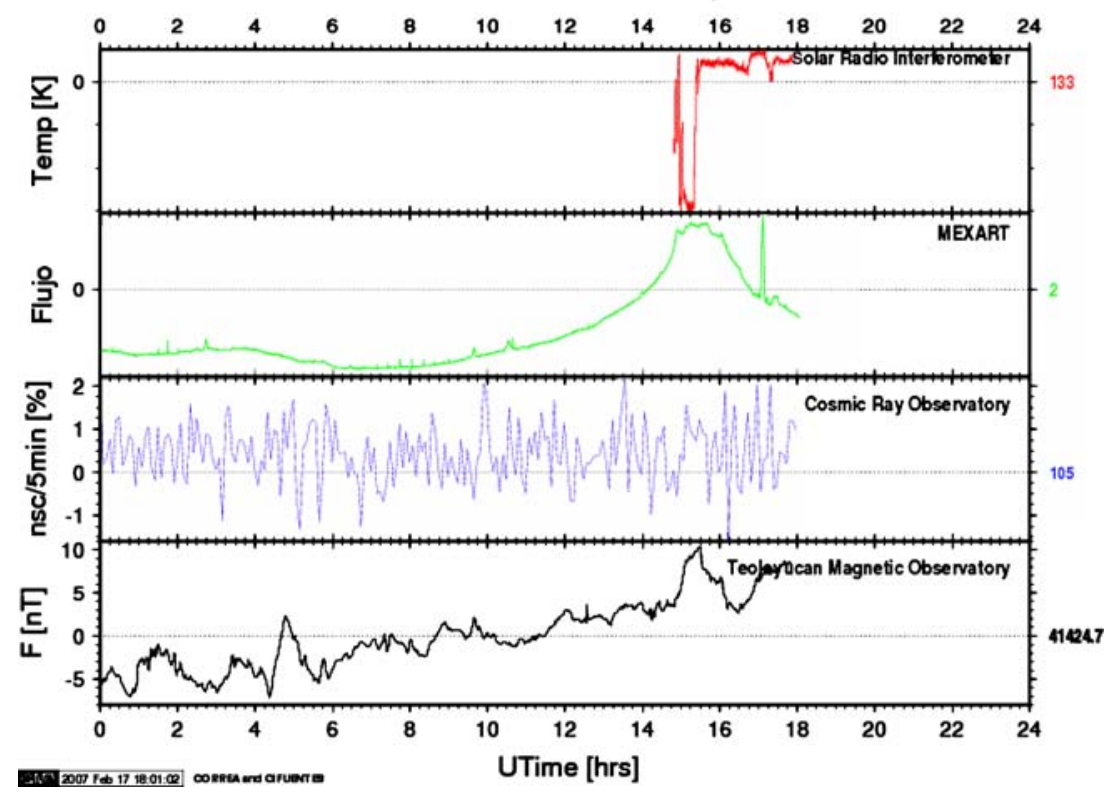


solar flare detected by RIS, there would be a halo CME propagating in the interplanetary medium which would affect the radio signals at MEXART, and the CR fluxes at RC. Depending on the velocity and magnetic configuration of the CME, this disturbance interacts with the Earth's magnetic field causing a geomagnetic storm detected by TEO. The VESO instruments puts together observations of four different phenomena associated with the space weather, allowing for qualitative and quantitative studies of a chain of events that start in the Sun and may affect the Earth's environment. The sequence may be followed starting from the triggering phenomenon at the Sun (RIS), make an estimate of the interplanetary consequences (MEXART and CR) and the eventual arrival at Earth (TEO).

At the moment the VESO database starts from February 19, 2007 (IHY official kick-off) but in the near future it will include all available data from the four observatories and facilities for specific times data searches. We want also to join scientific observations, with educational and outreach information in the same web server combining the objectives of the IHY and eGY celebrations.
Acknowledgments The MEXART measurements are operated by E. Andrade and A. Carrillo, and G. Casillas administrates the computing network. S. Jeyakumar and V. de la Luz wrote the MEXART programs to perform the real time data acquisition and plotting. The MEXART is partially funded by the PAPIIT IN100506-3 project.

\section{References}

Gonzalez-Esparza, Carrillo JA, Andrade E, Perez R, Kurtz S (2004) The MEXART interplanetary scintillation array in Mexico Geofis Int 43:161-73

Gonzalez-Esparza JA, Andrade E, Carrillo A, Jeyakumar S, Ananthakrishnan S, Praveenkumar A, Sankarasubramanian G, Sureshkumar S, Sierra P, Vazquez S, Perez-Enriquez R, Kurtz S (2005) MEXART measurements of radio sources, Proc. of the Solar Wind 11/SOHO 16 Conference "Connecting Sun and Heliosphere", B. Fleek and T.H. Zurbuchen editors, ESA SP-592, pp 667-669

Gonzales-Esparza JA, Andrade E, Carrillo A, Rodriguez C, PerezEnriquez R, Kurtz S, Blanco-Cano X (2006) Calibration testings of the MEXART antenna measuring solar transits. Adv Space Res 38:1824-1827

Harrison R, Breen A, Bromage B, Davila J (2005) 2007: International Heliophysical Year". Astron Geophys 46:3.27-3.30

Hatton CJ, Carmcheal H (1964) Can J Phys 1/12:2442

Rex D (2007) Geophysicists combine forces Nature 447:1037 